\title{
Periodic Solutions in UMD Spaces for Some Neutral Partial Functional Differential Equations
}

\author{
Rachid Bahloul'1, Khalil Ezzinbi², Omar Sidki ${ }^{1}$ \\ ${ }^{1}$ Département de Mathématiques, Faculté des Sciences et Technologie, Fès, Morocco \\ ${ }^{2}$ Département de Mathématiques, Faculté des Sciences Semlalia, Université Cadi Ayyad, Marrakech, Morocco \\ Email:bahloul33r@gmail.com, ezzinbi@uca.ac.ma, osidki@hotmail.com
}

How to cite this paper: Bahloul, R., Ezzinbi, K. and Sidki, O. (2016) Periodic Solutions in UMD Spaces for Some Neutral Partial Functional Differential Equations. Advances in Pure Mathematics, 6, 713-726. http://dx.doi.org/10.4236/apm.2016.610058

Received: February 23, 2016

Accepted: September 23, 2016

Published: September 26, 2016

Copyright $\odot 2016$ by authors and Scientific Research Publishing Inc. This work is licensed under the Creative Commons Attribution International License (CC BY 4.0). http://creativecommons.org/licenses/by/4.0/

\begin{abstract}
The aim of this work is to study the existence of a periodic solution for some neutral partial functional differential equations. Our approach is based on the R-boundedness of linear operators $L^{p}$-multipliers and UMD-spaces.
\end{abstract}

\section{Keywords}

Neutral Partial Functional Differential Equations, Periodic Solutions,

R-Boundedness, $L^{p}$-Multipliers, UMD Spaces

\section{Introduction}

Motivated by the fact that neutral functional differential equations (abbreviated, NFDE) with finite delay arise in many areas of applied mathematics, this type of equations has received much attention in recent years. In particular, the problem of existence of periodic solutions has been considered by several authors. We refer the readers to papers [1]-[8] and the references listed therein for information on this subject.

In this work, we study the existence of periodic solutions for the following neutral partial functional differential equations of the following form

$$
\frac{\mathrm{d}}{\mathrm{d} t}\left[x(t)-L\left(x_{t}\right)\right]=A\left[x(t)-L\left(x_{t}\right)\right]+G\left(x_{t}\right)+f(t) \text { for } t \in \mathbb{R},
$$

where $A: D(A) \subseteq X \rightarrow X$ is a linear closed operator on Banach space $(X,\|\|$.$) and$ $f \in L^{p}(\mathbb{T}, X)$ for all $p \geq 1$. For $r_{2 \pi}:=2 \pi N$ (some $N \in \mathbb{N}$ ) $L$ and $G$ are in $B\left(L^{p}\left(\left[-r_{2 \pi}, 0\right], X\right) ; X\right)$ is the space of all bounded linear operators and $x_{t}$ is an element of $L^{p}\left(\left[-r_{2 \pi}, 0\right], X\right)$ which is defined as follows

$$
x_{t}(\theta)=x(t+\theta) \text { for } \theta \in\left[-r_{2 \pi}, 0\right] .
$$


In [4], Ezzinbi et al. established the existence of periodic solutions for the following partial functional differential equation:

$$
\frac{\partial}{\partial t} w(x, t)=\frac{\partial^{2}}{\partial x^{2}} w(x, t)+b(t) \int_{-\infty}^{0} G(\theta) w(x+\theta, t) \mathrm{d} x+f(x, t),
$$

where $b: \mathbb{R} \rightarrow \mathbb{R}$ is a continuous $\omega$-periodic function, $f:[0, \pi] \times \mathbb{R} \rightarrow \mathbb{R}$ is a continuous function $\omega$-in $t$, periodic and $G$ is a positive function.

In [1], Arendt gave necessary and sufficient conditions for the existence of periodic solutions of the following evolution equation.

$$
\frac{\mathrm{d}}{\mathrm{d} t} x(t)=A x(t)+f(t) \text { for } t \in \mathbb{R},
$$

where $A$ is a closed linear operator on an UMD-space $Y$.

In [2], C. Lizama established results on the existence of periodic solutions of Equation (1) when $L=0$, namely, for the following partial functional differential equation

$$
\frac{\mathrm{d}}{\mathrm{d} t} x(t)=A x(t)+G\left(x_{t}\right)+f(t) \text { for } t \in \mathbb{R}
$$

where $(A, D(A))$ is a linear operator on an UMD-space $X$.

In [3], Hernan et al., studied the existence of periodic solution for the class of linear abstract neutral functional differential equation described in the following form:

$$
\frac{\mathrm{d}}{\mathrm{d} t}[x(t)-B x(t-r)]=A x(t)+G\left(x_{t}\right)+f(t) \text { for } t \in \mathbb{R}
$$

where $A: D(A) \rightarrow X$ and $B: D(B) \rightarrow X$ are closed linear operator such that $D(A) \subset D(B)$ and $G \in B\left(L^{p}([-2 \pi, 0], X) ; X\right)$.

The organisation of this work is as follows: In Section 2, we present preliminary results on UMD spaces. In Section 3, we study the existence of periodic strong solution for Equation (1) with finite delay and we discuss the existence of mild solutions of Equation (1). In Section 4, we give the main abstract result [Theorem 4.1] of this work, and some important consequence when $A$ generates a $C_{0}$-semigroup [Theorem 4.2]. The last section is devoted to some examples.

\section{UMD Spaces}

Let $X$ be a Banach space. Firstly, we denote By $\mathbb{T}$ the group defined as the quotient $\mathbb{R} / 2 \pi \mathbb{Z}$. There is an identification between functions on $\mathbb{T}$ and $2 \pi$-periodic functions on $\mathbb{R}$. We consider the interval $[0,2 \pi)$ as a model for $\mathbb{T}$.

Given $1 \leq p<\infty$, we denote by $L^{p}(\mathbb{T} ; X)$ the space of $2 \pi$-periodic locally $p$-integrable functions from $\mathbb{R}$ into $X$, with the norm:

$$
\|f\|_{p}:=\left(\int_{0}^{2 \pi}\|f(t)\|^{p} \mathrm{~d} t\right)^{1 / p}
$$

For $f \in L^{p}(\mathbb{T} ; X)$, we denote by $\hat{f}(k), k \in \mathbb{Z}$ the $k$-th Fourier coefficient of $f$ that is defined by:

$$
\hat{f}(k)=\frac{1}{2 \pi} \int_{0}^{2 \pi} \mathrm{e}^{-i k t} f(t) \mathrm{d} t \text { for } k \in \mathbb{Z} \text { and } t \in \mathbb{R} \text {. }
$$


Definition 2.1 Let $\varepsilon \in] 0,1\left[\right.$ and $1<p<\infty$. Define the operator $H_{\varepsilon}$ by: for all $f \in L^{p}(\mathbb{R} ; X)$

$$
\left(H_{\varepsilon} f\right)(t):=\frac{1}{\pi} \int_{\varepsilon<|s|<\frac{1}{\varepsilon}} \frac{f(t-s)}{s} \mathrm{~d} s
$$

if $\lim H_{\varepsilon} f:=H f$ exists in $L^{p}(\mathbb{R} ; X)$ Then, $H f$ is called the Hilbert transform of $f$ on $\stackrel{\varepsilon \rightarrow 0}{L^{p}}(\mathbb{R} ; X)$.

Definition 2.2 [2]

A Banach space $X$ is said to be UMD space if the Hilbert transform is bounded on $L^{p}(\mathbb{R} ; X)$ for all $1<p<\infty$.

Example 2.1 [9] 1) Any Hilbert space is an UMD space.

2) $L^{p} \quad$ (0.1) are UMD spaces for every $1<p<\infty$.

3) Any closed subspace of UMD space is an UMD space.

\section{$R$-Bounded and $L^{p-M u l t i p l i e r s}$}

Let $X$ and $Y$ be Banach spaces. Then $B(X, Y)$ denotes the space of bounded linear operators from $X$ to $Y$.

Definition 2.3 [1]

A family of operators $T=\left(T_{j}\right)_{j \in \mathbb{N}^{*}} \subset B(X, Y)$ is called R-bounded (Rademacher bounded or randomized bounded $)$, if there is a constant $C>0$ and $p \in[1, \infty)$ such that for each $n \in N, T_{j} \in T, \quad x_{j} \in X$ and for all independent, symmetric, $\{-1,1\}$-valued random variables $r_{j}$ on a probability space $(\Omega, M, \mu)$ the inequality

$$
\left\|\sum_{j=1}^{n} r_{j} T_{j} x_{j}\right\|_{L^{p}(0,1 ; Y)} \leq C\left\|\sum_{j=1}^{n} r_{j} x_{j}\right\|_{L^{p}(0,1 ; X)}
$$

is valid. The smallest $C$ is called $R$-bounded of $\left(T_{j}\right)_{j \in \mathbb{N}^{*}}$ and it is denoted by $R_{p}(T)$.

Lemma 2.1 ([2], Remark 2.2)

1) If $T=\left(T_{j}\right)_{j \in \mathbb{N}^{*}} \subset B(X, Y)$ is $R$-bounded then it is uniformly bounded, with

$$
\sup \left\{\left\|T_{j}\right\|: T_{j} \in T\right\} \leq R_{p}(T) .
$$

2) The definition of $R$-boundedness is independent of $p \in[1, \infty)$.

Definition 2.4 [1] For $1 \leq p<\infty$, a sequence $\left\{M_{k}\right\}_{k \in \mathbb{Z}} \subset \mathbf{B}(X, Y)$ is said to be an $L^{p}$-multiplier if for each $f \in L^{p}(\mathbb{T}, X)$, there exists $u \in L^{p}(\mathbb{T}, Y)$ such that $\hat{u}(k)=M_{k} \hat{f}(k)$ for all $k \in \mathbb{Z}$.

Proposition 2.1 ([1], Proposition 1.11) Let X be a Banach space and $\left\{M_{k}\right\}_{k \in \mathbb{Z}}$ be an $L^{p}$-multiplier, where $1 \leq p<\infty$. Then the set $\left\{M_{k}\right\}_{k \in \mathbb{Z}}$ is $R$-bounded.

Theorem 2.1 (Marcinkiewicz operator-valud multiplier Theorem).

Let $X, Y$ be UMD spaces and $\left\{M_{k}\right\}_{k \in \mathbb{Z}} \subset B(X, Y)$. If the sets $\left\{M_{k}\right\}_{k \in \mathbb{Z}}$ and $\left\{k\left(M_{k+1}-M_{k}\right)\right\}_{k \in \mathbb{Z}}$ are $R$-bounded, then $\left\{M_{k}\right\}_{k \in \mathbb{Z}}$ is an $L^{p}$-multiplier for $1<p<\infty$.

Theorem 2.2 [2] Let $f \in L^{p}(\mathbb{T}, X)$. Then

$$
f=\lim _{n \rightarrow \infty} \sigma_{n}(f)
$$

in $L^{p}(\mathbb{T}, X)$ where 


$$
\sigma_{n}(f):=\frac{1}{n+1} \sum_{m=0}^{n} \sum_{k=-m}^{m} e_{k} \hat{f}(k)
$$

with $e_{k}(t):=\mathrm{e}^{i k t}$.

Theorem 2.3 (Neumann Expansion) Let $A \in B(X, X)$, where $X$ is a Banach space. If $\|A\|<1$ then $I-A$ is invertible, moreover

$$
(I-A)^{-1}=\sum_{k=0}^{\infty} A^{k}
$$

\section{Periodic Solutions for Equation (1)}

Lemma 3.1 Let $f \in L^{1}(\mathbb{T} ; X)$. If $g(t)=\int_{0}^{t} f(s) \mathrm{d} s$ and $k \in \mathbb{Z}, k \neq 0$. Then

$$
\hat{g}(k)=\frac{i}{k} \hat{f}(0)-\frac{i}{k} \hat{f}(k) .
$$

Proof. Let $g(t)=\int_{0}^{t} f(s)$ ds. Then by applying the Fourier transform, we obtain that

$$
\hat{g}(k)=\frac{1}{2 \pi} \int_{0}^{2 \pi} \mathrm{e}^{-i k t} g(t) \mathrm{d} t=\frac{1}{2 \pi} \int_{0}^{2 \pi} \mathrm{e}^{-i k t} \int_{0}^{t} f(s) \mathrm{d} s \mathrm{~d} t .
$$

Integration by parts we obtain that

$$
\begin{aligned}
\hat{g}(k) & =\frac{1}{2 \pi}\left[\frac{\mathrm{e}^{-i k t}}{-i k} \int_{0}^{t} f(s) \mathrm{d} s\right]_{0}^{2 \pi}+\frac{1}{2 \pi} \int_{0}^{2 \pi} \frac{1}{i k} \mathrm{e}^{-i k t} f(t) \mathrm{d} t \\
& =\frac{1}{-i k} \hat{f}(0)+\frac{1}{i k} \hat{f}(k)=\frac{i}{k} \hat{f}(0)-\frac{i}{k} \hat{f}(k) .
\end{aligned}
$$

The proof is complete.

Lemma 3.2 [1] Let $1 \leq p<\infty$ and $u, v \in L^{p}(\mathbb{T} ; X)$. Then the following assertions are equivalent.

1) $\int_{0}^{2 \pi} v(s) \mathrm{d} s=0$ and there exists $x \in X$ such that

$$
u(t)=x+\int_{0}^{t} v(s) \mathrm{d} s
$$

2) $\hat{v}(k)=i k \hat{u}(k)$ for any $k \in \mathbb{Z}$.

Let

$$
H^{1, p}(\mathbb{T} ; X)=\left\{u \in L^{p}(\mathbb{T}, X): \exists v \in L^{p}(\mathbb{T}, X), \hat{v}(k)=i k \hat{u}(k) \text { for all } k \in \mathbb{Z}\right\} .
$$

By a Lemma 3.2 we obtain that

$(\alpha): u \in H^{1, p}(\mathbb{T} ; X) \Leftrightarrow u \in L^{p}(\mathbb{T} ; X), \exists v \in L^{p}(\mathbb{T} ; X)$ such that $\int_{0}^{2 \pi} v(s) \mathrm{d} s=0$ and there exists $x \in X$ with $u(t)=x+\int_{0}^{t} v(s) \mathrm{d} s$.

Definition 3.1 [2]. For $1 \leq p<\infty$, we say that a sequence $\left\{M_{k}\right\}_{k \in \mathbb{Z}} \subset \mathbf{B}(X, Y)$ is an $\left(L^{p}, H^{1, p}\right)$-multiplier, if for each $f \in L^{p}(\mathbb{T}, X)$ there exists $u \in H^{1, p}(\mathbb{T}, Y)$ such that

$$
\hat{u}(k)=M_{k} \hat{f}(k) \text { for all } k \in \mathbb{Z} \text {. }
$$

Lemma 3.3 [2] Let $1 \leq p<\infty$ and $\left(M_{k}\right)_{k \in \mathbb{Z}} \subset \mathbf{B}(X) \quad(\mathbf{B}(X)$ is the set of all bounded linear operators from $X$ to $X$ ). Then the following assertions are equivalent. 
1) $\left(M_{k}\right)_{k \in \mathbb{Z}}$ is an $\left(L^{p}, H^{1, p}\right)$-multiplier.

2) $\left(i k M_{k}\right)_{k \in \mathbb{Z}}$ is an $\left(L^{p}, L^{p}\right)$-multiplier.

\subsection{Existence of Strong Solutions for Equation (2)}

Let $D \varphi=\varphi(0)-L(\varphi)$.

Then the Equation (1) is equivalent:

$$
\frac{\mathrm{d}}{\mathrm{d} t}\left(D x_{t}\right)=A\left(D x_{t}\right)+G\left(x_{t}\right)+f(t) \text { for } t \in \mathbb{R} .
$$

Denote by $L_{k}(x):=L\left(e_{k} x\right) ; G_{k}(x):=G\left(e_{k} x\right)$ and $e_{k}(\theta):=\mathrm{e}^{i k \theta}, D_{k}=I-L_{k}$ for all $k \in \mathbb{Z}$. We define

$$
\Delta_{k}=\left(i k D_{k}-A D_{k}-G_{k}\right) \text { and } \sigma_{\mathbb{Z}}(\Delta)=\left\{k \in \mathbb{Z}: \Delta_{k} \text { is not bijective }\right\} .
$$

We begin by establishing our concept of strong solution for Equation (2).

Definition 3.2 Let $f \in L^{p}(\mathbb{T} ; X)$. A function $x \in H^{1, p}(\mathbb{T} ; X)$ is said to be a $2 \pi$ periodic strong $L^{p}$-solution of Equation (2) if $D x_{t} \in D(A)$ for all $t \geq 0$ and Equation (2) holds almost every where.

Lemma 3.4 Let $G: L^{p}(\mathbb{T}, X) \rightarrow X$ be a bounded linear operateur. Then

$$
\widehat{G(u)}(k)=G\left(e_{k} \hat{u}(k)\right):=G_{k} \hat{u}(k) \text { for all } k \in \mathbb{Z} \text {. }
$$

Proof. Let $\theta \in\left[-r_{2 \pi}, 0\right]$. Then

$$
\begin{aligned}
\left(e_{k} \hat{u}(k)\right)(\theta)= & \frac{1}{2 \pi} \int_{0}^{2 \pi} \mathrm{e}^{i k(\theta-s)} u(s) \mathrm{d} s \\
= & \frac{1}{2 \pi} \int_{0}^{2 \pi} \mathrm{e}^{-i k(s-\theta)} u(s) \mathrm{d} s \\
= & \frac{1}{2 \pi} \int_{-\theta}^{2 \pi-\theta} \mathrm{e}^{-i k s^{\prime}} u\left(s^{\prime}+\theta\right) \mathrm{d} s,\left(s^{\prime}=s-\theta\right) \\
= & \frac{1}{2 \pi} \int_{-\theta}^{0} \mathrm{e}^{-i k s^{\prime}} u\left(s^{\prime}+\theta\right) \mathrm{d} s^{\prime}+\frac{1}{2 \pi} \int_{0}^{2 \pi} \mathrm{e}^{-i k s^{\prime}} u\left(s^{\prime}+\theta\right) \mathrm{d} s^{\prime} \\
& +\frac{1}{2 \pi} \int_{2 \pi}^{2 \pi-\theta} \mathrm{e}^{-i k s^{\prime}} u\left(s^{\prime}+\theta\right) \mathrm{d} s^{\prime} .
\end{aligned}
$$

Moreover

$$
\begin{aligned}
& \frac{1}{2 \pi} \int_{2 \pi}^{2 \pi-\theta} \mathrm{e}^{-i k s^{\prime}} u\left(s^{\prime}+\theta\right) \mathrm{d} s^{\prime} \\
& =\frac{1}{2 \pi} \int_{0}^{-\theta} \mathrm{e}^{-i k(2 \pi-t)} u(2 \pi-t+\theta) \mathrm{d} t,(t=2 \pi-s) \\
& =\frac{1}{2 \pi} \int_{0}^{-\theta} \mathrm{e}^{i k t} u(-t+\theta) \mathrm{d} t,(u \text { is } 2 \pi \text {-periodic }) \\
& =\frac{1}{2 \pi} \int_{0}^{\theta} \mathrm{e}^{-i k s} u(s+\theta) \mathrm{d} t,(-t=s) .
\end{aligned}
$$

It follows

$$
\left(e_{k} \hat{u}(k)\right)(\theta)=\frac{1}{2 \pi} \int_{0}^{2 \pi} \mathrm{e}^{-i k(s-\theta)} u(s) \mathrm{d} s=\frac{1}{2 \pi} \int_{0}^{2 \pi} \mathrm{e}^{-i k s} u_{s}(\theta) \mathrm{d} s .
$$

Since $G$ is bounded, then 


$$
G\left(e_{k} \hat{u}(k)\right)=G\left(\frac{1}{2 \pi} \int_{0}^{2 \pi} \mathrm{e}^{-i k s} u_{s} \mathrm{~d} s\right)=\frac{1}{2 \pi} \int_{0}^{2 \pi} \mathrm{e}^{-i k s} G u_{s} \mathrm{~d} s .
$$

Then

$$
G\left(e_{k} \hat{u}(k)\right)=\widehat{G(u)}(k) \text { i.e } G_{k} \hat{u}(k)=\widehat{G(u)}(k)
$$

Lemma 3.5 [1] Let $X$ be a Banach space, $n \in \mathbb{N}, x_{j} \in X, r_{j}$ independent, symmetric, $\{-1,1\}$-valued random variables on a probability space $(\Omega, M, \mu)$, and $\alpha_{j}, \beta_{j} \in \mathbb{C}$ such that $\left|\alpha_{j}\right| \leq\left|\beta_{j}\right|$, for each $j=1, \cdots, n$. Then

$$
\left\|\sum_{j=1}^{n} \alpha_{j} r_{j} x_{j}\right\|_{L^{p}(0,1 ; X)}^{p} \leq 2\left\|\sum_{j=1}^{n} \beta_{j} r_{j} x_{j}\right\|_{L^{p}(0,1 ; X)}^{p} .
$$

Proposition 3.1 Let $A$ be a closed linear operator defined on an UMD space $X$. Suppose that $\sigma_{\mathbb{Z}}(\Delta)=\phi$. Then the following assertions are equivalent.

1) $\left(i k\left(i k D_{k}-A D_{k}-G_{k}\right)^{-1}\right)_{k \in \mathbb{Z}}$ is an $L^{p}$-multiplier for $1<p<\infty$

2) $\left(i k\left(i k D_{k}-A D_{k}-G_{k}\right)^{-1}\right)_{k \in \mathbb{Z}}$ is $R$-bounded.

Proof. 1) $\Rightarrow$ 2) As a consequence of Proposition 2.1

$2) \Rightarrow 1$ ) We claim first that the set $\{G\}_{k \in \mathbb{Z}}$ is $R$-bounded. In fact, for $x_{j} \in D(A)$ we have:

$$
\begin{aligned}
\left\|\sum_{j=1}^{n} r_{j} G_{j} x_{j}\right\|_{L^{p}(0,1 ; X)}^{p} & =\int_{0}^{1}\left\|\sum_{j=1}^{n} r_{j}(t) G\left(e_{j} x_{j}\right)\right\|_{X}^{p} \mathrm{~d} t \\
& =\int_{0}^{1}\left\|G\left(\sum_{j=1}^{n} r_{j}(t) e_{j} x_{j}\right)\right\|_{X}^{p} \mathrm{~d} t \\
& \leq\|G\|^{p} \int_{0}^{1}\left\|\sum_{j=1}^{n} r_{j}(t) e_{j} x_{j}\right\|_{L^{p}\left(\left[-r_{2 \pi}, 0\right] ; x\right)}^{p} \mathrm{~d} t \\
& \leq\|G\|^{p} \int_{0}^{1} \int_{-r_{2 \pi}}^{0}\left\|\sum_{j=1}^{n} r_{j}(t) e_{j}(s) x_{j}\right\|_{X}^{p} \mathrm{~d} s \mathrm{~d} t \\
& \leq\|G\|^{p} \int_{-r_{2 \pi}}^{0} \int_{0}^{1}\left\|\sum_{j=1}^{n} r_{j}(t) e_{j}(s) x_{j}\right\|_{X}^{p} \mathrm{~d} t \mathrm{~d} s .
\end{aligned}
$$

Since

$$
\int_{0}^{1}\left\|\sum_{j=1}^{n} r_{j}(t) e_{j}(s) x_{j}\right\|_{X}^{p} \mathrm{~d} t=\left\|\sum_{j=1}^{n} r_{j} e_{j}(s) x_{j}\right\|_{L^{p}(0,1 ; X)}^{p}
$$

Then

$$
\left\|\sum_{j=1}^{n} r_{j} G_{j} x_{j}\right\|_{L^{p}(0,1 ; X)}^{p} \leq\|G\|^{p} \int_{-r_{2 \pi} \pi}^{0}\left\|\sum_{j=1}^{n} r_{j} e_{j}(s) x_{j}\right\|_{L^{p}(0,1 ; X)}^{p} \mathrm{~d} s .
$$

By Lemma 3.4, we obtain that

$$
\left\|\sum_{j=1}^{n} r_{j} G_{j} x_{j}\right\|_{L^{p}(0,1 ; X)}^{p} \leq 2\|G\|^{p} \int_{-r_{2 \pi}}^{0}\left\|\sum_{j=1}^{n} r_{j} x_{j}\right\|_{L^{p}(0,1 ; X)}^{p} \mathrm{~d} s \leq 2 r_{2 \pi}\|G\|^{p}\left\|\sum_{j=1}^{n} r_{j} x_{j}\right\|_{L^{p}(0,1 ; X)}^{p} .
$$

We conclude that 


$$
R_{p}\left(\left(G_{k}\right)_{k \in \mathbb{Z}}\right) \leq\left(2 r_{2 \pi}\right)^{1 / p}\|G\| .
$$

Next define $M_{k}=i k\left(C_{k}-A D_{k}\right)^{-1}$, where $C_{k}:=i k D_{k}-G_{k}$. By Theorem 2.1 it is sufficient to prove that the set $\left\{k\left(M_{k+1}-M_{k}\right)\right\}_{k \in \mathbb{Z}}$ is $R$-bounded. Since

$$
\begin{aligned}
k & {\left[M_{k+1}-M_{k}\right]=k\left[i(k+1)\left(C_{k+1}-A D_{k+1}\right)^{-1}-i k\left(C_{k}-A D_{k}\right)^{-1}\right] } \\
= & k\left(C_{k+1}-A D_{k+1}\right)^{-1}\left[i(k+1)\left(C_{k}-A D_{k}\right)-i k\left(C_{k+1}-A D_{k+1}\right)\right]\left(C_{k}-A D_{k}\right)^{-1} \\
= & k\left(C_{k+1}-A D_{k+1}\right)^{-1}\left[i k\left(C_{k}-C_{k+1}\right)+i\left(C_{k}-A D_{k}\right)+i k\left(A D_{k+1}-A D_{k}\right)\right]\left(C_{k}-A D_{k}\right)^{-1} \\
= & k\left(C_{k+1}-A D_{k+1}\right)^{-1}\left[i k\left(C_{k}-C_{k+1}\right)\left(C_{k}-A D_{k}\right)^{-1}+i+i k\left(A D_{k+1}-A D_{k}\right)\left(C_{k}-A D_{k}\right)^{-1}\right] \\
= & k\left(C_{k+1}-A D_{k+1}\right)^{-1}\left(C_{k}-C_{k+1}\right) i k\left(C_{k}-A D_{k}\right)^{-1}+i k\left(C_{k+1}-A D_{k+1}\right)^{-1} \\
& +k\left(C_{k+1}-A D_{k+1}\right)^{-1}\left(A D_{k+1}-A D_{k}\right) i k\left(C_{k}-A D_{k}\right)^{-1}
\end{aligned}
$$

we have

$$
\begin{aligned}
C_{k}-C_{k+1} & =i k D_{k}-i(k+1) D_{k+1}+G_{k+1}-G_{k} \\
& =i k\left(D_{k}-D_{k+1}\right)-i D_{k+1}+\left(G_{k+1}-G_{k}\right) \\
& =i k\left(L_{k+1}-L_{k}\right)+\left(G_{k+1}-G_{k}\right)+i L_{k}-i I .
\end{aligned}
$$

Therefore

$$
\begin{aligned}
k & \left(C_{k+1}-A D_{k+1}\right)^{-1}\left(A D_{k+1}-A D_{k}\right) i k\left(C_{k}-A D_{k}\right)^{-1} \\
= & k\left(C_{k+1}-A D_{k+1}\right)^{-1} A D_{k+1} i k\left(C_{k}-A D_{k}\right)^{-1} \\
& -k\left(C_{k+1}-A D_{k+1}\right)^{-1} i k A D_{k}\left(C_{k}-A D_{k}\right)^{-1} \\
= & k\left[C_{k+1}\left(C_{k+1}-A D_{k+1}\right)^{-1}+I\right] i k\left(C_{k}-A D_{k}\right)^{-1} \\
& -k\left(C_{k+1}-A D_{k+1}\right)^{-1} i k\left[I-C_{k}\left(C_{k}-A D_{k}\right)^{-1}\right] .
\end{aligned}
$$

Since products and sums of $R$-bounded sequences is $R$-bounded [10. Remark 2.2]. Then the proof is complete.

Lemma 3.6 Let $1 \leq p<\infty$. Suppose that $\sigma_{\mathbb{Z}}(\Delta)=\phi$ and that for every $f \in L^{p}(\mathbb{T} ; X)$ there exists a $2 \pi$-periodic strong $L^{p}$-solution $x$ of Equation (2). Then, $X$ is the unique $2 \pi$-periodic strong $L^{p}$-solution.

Proof. Suppose that $x_{1}$ and $x_{2}$ two strong $L^{p}$-solution of Equation (2) then $x=x_{1}-x_{2}$ is a strong $L^{p}$-solution of Equation (2) corresponding to $f=0$. Taking Fourier transform in (2), we obtain that

$$
i k D_{k} \hat{x}(k)=A D_{k} \hat{x}(k)+G_{k} \hat{x}(k), k \in \mathbb{Z} .
$$

Then

$$
\left(i k D_{k}-A D_{k}-G_{k}\right) \hat{x}(k)=0 \text {. }
$$

It follows that $\hat{x}(k)=0$ for every $k \in \mathbb{Z}$ and therefore $x=0$. Then $x_{1}=x_{2}$.

Theorem 3.1 Let $X$ be a Banach space. Suppose that for every $f \in L^{p}(\mathbb{T} ; X)$ there exists a unique strong solution of Equation (2) for $1 \leq p<\infty$. Then 
1) for every $k \in \mathbb{Z}$ the operator $\Delta_{k}=\left(i k D_{k}-A D_{k}-G_{k}\right)$ has bounded inverse

2) $\left\{i k \Delta_{k}^{-1}\right\}_{k \in \mathbb{Z}}$ is $R$-bounded.

Before to give the proof of Theorem 3.1, we need the following Lemma.

Lemma 3.7 if $\left(i k D_{k}-A D_{k}-G_{k}\right)(x)=0$ for all $k \in \mathbb{Z}$, then $u_{t}()=.\mathrm{e}^{i k t} e_{k}()$.$x is a$ $2 \pi$-periodic strong $L^{p}$-solution of the following equation

$$
\frac{\mathrm{d}}{\mathrm{d} t}\left(D x_{t}\right)=A\left(D x_{t}\right)+G x_{t}
$$

Proof of Lemma $3.7\left(i k D_{k}-A D_{k}-G_{k}\right)(x)=0 \Rightarrow i k D_{k} x=A D_{k} x+G_{k} x$.

Then

$$
i k x=i k L_{k} x+A D_{k} x+G_{k} x
$$

We have $u_{t}=\mathrm{e}^{i k t} e_{k} X$ and

$$
\begin{aligned}
u_{t}^{\prime}= & i k \mathrm{e}^{i k t} e_{k} x=\mathrm{e}^{i k t} e_{k}(i k x) \\
= & \mathrm{e}^{i k t} e_{k}\left[i k L_{k} x+A D_{k} x+G_{k} x\right] \\
= & i k \mathrm{e}^{i k t} e_{k} L_{k} x+\mathrm{e}^{i k t} e_{k} A D_{k} x+\mathrm{e}^{i k t} e_{k} G_{k} x \\
= & i k L\left(\mathrm{e}^{i k t} e_{k} x\right)+A D\left(\mathrm{e}^{i k t} e_{k} x\right)+G\left(\mathrm{e}^{i k t} e_{k} x\right) \\
= & i k L\left(u_{t}\right)+A\left(D u_{t}\right)+G\left(u_{t}\right) \\
= & \left(L u_{t}\right)^{\prime}+A\left(D u_{t}\right)+G\left(u_{t}\right) \\
& \quad\left(u_{t}-L u_{t}\right)^{\prime}=A\left(D u_{t}\right)+G\left(u_{t}\right) \\
& \left(D u_{t}\right)^{\prime}=A\left(D u_{t}\right)+G\left(u_{t}\right) .
\end{aligned}
$$

Proof of Theorem 3.1:1) Let $k \in \mathbb{Z}$ and $y \in X$. Then for $f(t)=\mathrm{e}^{i k t} y$, there exists $x \in H^{1, p}(\mathbb{T} ; X)$ such that:

$$
\frac{\mathrm{d}}{\mathrm{d} t} D x_{t}=A\left(D x_{t}\right)+G\left(x_{t}\right)+f(t) .
$$

Taking Fourier transform, $G$ and $D$ are bounded. We have

$\widehat{(D x)^{\prime}}(k)=\widehat{x^{\prime}}(k)-\widehat{(L x)^{\prime}}(k)$ by Lemma 3.2 and Lemma 3.4, we deduce that:

$$
\widehat{x^{\prime}}(k)-\widehat{(L x)^{\prime}}(k)=i k \hat{x}(k)-i k L_{k} \hat{x}(k)=i k\left(I-L_{k}\right) \hat{x}(k)=i k D_{k} \hat{x}(k) .
$$

Consequently, we have

$$
i k D_{k} \hat{x}(k)=A D_{k} \hat{x}(k)+G_{k} \hat{x}(k)+\hat{f}(k)
$$

$\left(i k D_{k}-A D_{k}-G_{k}\right) \hat{x}(k)=\hat{f}(k)=y \Rightarrow\left(i k D_{k}-A D_{k}-G_{k}\right)$ is surjective.

If $\left(i k D_{k}-A D_{k}-G_{k}\right)(u)=0$, then by Lemma 3.7, $x_{t}=\mathrm{e}^{i k t} e_{k} u$ is a $2 \pi$-periodic strong $L^{p}$-solution of Equation (2) corresponing to the function $f(t)=0$ Hence $x_{t}=0$ and $u=0$ then $\left(i k D_{k}-A D_{k}-G_{k}\right)$ is injective.

2) Let $f \in L^{p}(\mathbb{T} ; X)$. By hypothesis, there exists a unique $x \in H^{1, p}(\mathbb{T}, X)$ such that the Equation (2) is valid. Taking Fourier transforms, we deduce that

$$
\hat{x}(k)=\left(i k D_{k}-A D_{k}-G_{k}\right)^{-1} \hat{f}(k) \text { for all } k \in \mathbb{Z} \text {. }
$$


Hence

$$
i k \hat{x}(k)=i k\left(i k D_{k}-A D_{k}-G_{k}\right)^{-1} \hat{f}(k) \text { for all } k \in \mathbb{Z} \text {. }
$$

Since $x \in H^{1, p}(\mathbb{T} ; X)$, then there exists $v \in L^{p}(\mathbb{T} ; X)$ such that

$$
\hat{v}(k)=i k \hat{x}(k)=i k\left(i k D_{k}-A D_{k}-G_{k}\right)^{-1} \hat{f}(k) .
$$

Then $\left\{i k \Delta_{k}^{-1}\right\}_{k \in \mathbb{Z}}$ is an $L^{p}$-multiplier and $\left\{i k \Delta_{k}^{-1}\right\}_{k \in \mathbb{Z}}$ is $R$-bounded.

\subsection{Periodic Mild Solutions of Equation (2) When $A$ Generates a $C_{0}$-Semigroup}

It is well known that in many important applications the operator $A$ can be the infinitesimal generator of $C_{0}$-semigroup $(T(t))_{t \geq 0}$ on the space $X$.

Definition 3.3 Assume that $A$ generates a $C_{0}$-semigroup $(T(t))_{t \geq 0}$ on $X$. A function $x$ is called a mild solution of Equation (2) if.

$$
D x_{t}=T(t) D \varphi+\int_{0}^{t} T(t-s)\left(G x_{s}+f(s)\right) \mathrm{d} s \text { for } 0 \leq t \leq 2 \pi .
$$

Remark 3.1 ([3], Remark 4.2) Let $(T(t))_{t \geq 0}$ be the $C_{0}$-semigroup generated by $A$. If $g:[0, a] \rightarrow X$ is a continuous function, then $\int_{0}^{t} \int_{0}^{s} T(t-s) g(\xi) \mathrm{d} \xi \mathrm{d} s \in D(A)$ and

$$
A \int_{0}^{t} \int_{0}^{s} T(t-s) g(\xi) \mathrm{d} \xi \mathrm{d} s=\int_{0}^{t}(T(t-s)-I) g(s) \mathrm{d} s \text { for all } 0 \leq t \leq a .
$$

Lemma 3.8 [3] Assume that $A$ generates a $C_{0}$-semigroup $\{T(t)\}_{t \geq 0}$ on $X$, if $X$ is a mild solution then

$$
D x_{t}=D \varphi+A \int_{0}^{t} D x_{s} \mathrm{~d} s+\int_{0}^{t}\left(G x_{s}+f(s)\right) \mathrm{d} s \text { for } 0 \leq t \leq 2 \pi .
$$

Theorem 3.2 Assume that A generates a $C_{0}$-semigroup $(T(t))_{t \geq 0}$ on $X$ and $f \in L^{p}(\mathbb{T} ; X)$. For some $1 \leq p<\infty$; if $X$ is a mild solution of Equation (2). Then

$$
\left(i k D_{k}-A D_{k}-G_{k}\right) \hat{x}(k)=\hat{f}(k) \text { for all } k \in \mathbb{Z} \text {. }
$$

Proof. Let $x$ be a mild solution of Equation (2). Then by Lemma 3.8, we have

$$
D x_{t}=D \varphi+A \int_{0}^{t} D x_{s} \mathrm{~d} s+\int_{0}^{t}\left(G x_{s}+f(s)\right) \mathrm{d} s .
$$

For $t=2 \pi$, we have

$$
D x_{2 \pi}=D \varphi+A \int_{0}^{2 \pi} D x_{s} \mathrm{~d} s+\int_{0}^{2 \pi}\left(G x_{s}+f(s)\right) \mathrm{d} s .
$$

Since: $D x_{2 \pi}=D \varphi$, then

$$
\begin{gathered}
A \int_{0}^{2 \pi} D x_{s} \mathrm{~d} s+\int_{0}^{2 \pi}\left(G x_{s}+f(s)\right) \mathrm{d} s=0 \\
\Rightarrow \frac{1}{2 \pi} A \int_{0}^{2 \pi} D x_{s} \mathrm{~d} s+\frac{1}{2 \pi} \int_{0}^{2 \pi}\left(G x_{s}+f(s)\right) \mathrm{d} s=0 \\
\Rightarrow \frac{1}{2 \pi} A \int_{0}^{2 \pi}\left(x(s)-L x_{s}\right) \mathrm{d} s+\frac{1}{2 \pi} \int_{0}^{2 \pi} \mathrm{e}^{-i 0 s} G x_{s} \mathrm{~d} s+\frac{1}{2 \pi} \int_{0}^{2 \pi} \mathrm{e}^{-i 0 s} f(s) \mathrm{d} s=0 \\
\Rightarrow \frac{1}{2 \pi} A \int_{0}^{2 \pi} \mathrm{e}^{-i 0 s}\left(x(s)-L x_{s}\right) \mathrm{d} s+\widehat{(G x)}(0)+\hat{f}(0)=0
\end{gathered}
$$




$$
\begin{gathered}
\Rightarrow A\left(\hat{x}(0)-L_{0} \hat{x}(0)\right)+G_{0} \hat{x}(0)+\hat{f}(0)=0 \\
\Rightarrow A D_{0} \hat{x}(0)+G_{0} \hat{x}(0)+\hat{f}(0)=0 \\
\Rightarrow\left(A D_{0}+G_{0}\right) \hat{x}(0)+\hat{f}(0)=0 \\
\Rightarrow\left(0-A D_{0}-G_{0}\right) \hat{x}(0)=\hat{f}(0),
\end{gathered}
$$

which shows that the assertion holds for $k=0$.

Now, define $v(t)=\int_{0}^{t} D x_{s} \mathrm{~d} s$ and $g(t)=D x_{t}-D \phi-\int_{0}^{t}\left(G x_{s}+f(s)\right) \mathrm{d} s$ by Lemma 3.1 We have:

$$
\begin{gathered}
\hat{v}(k)=\frac{i}{k} D_{0} \hat{x}(0)-\frac{i}{k} D_{k} \hat{x}(k) \\
A \hat{v}(k)=\frac{i}{k} A D_{0} \hat{x}(0)-\frac{i}{k} A D_{k} \hat{x}(k) \\
\hat{g}(k)=D_{k} \hat{x}(k)-\left[\frac{i}{k} G_{0} \hat{x}(0)-\frac{i}{k} G_{k} \hat{x}(k)\right]-\left[\frac{i}{k} \hat{f}(0)-\frac{i}{k} \hat{f}(k)\right] \\
=D_{k} \hat{x}(k)-\frac{i}{k} G_{0} \hat{x}(0)+\frac{i}{k} G_{k} \hat{x}(k)-\frac{i}{k} \hat{f}(0)+\frac{i}{k} \hat{f}(k) .
\end{gathered}
$$

Then

$$
\begin{gathered}
i k D_{k} \hat{x}(k)+G_{0} \hat{x}(0)-G_{k} \hat{x}(k)+\hat{f}(0)-\hat{f}(k)=-A D_{0} \hat{x}(0)+A D_{k} \hat{x}(k) \\
\Leftrightarrow\left[i k D_{k} \hat{x}(k)-A D_{k} \hat{x}(k)-G_{k} \hat{x}(k)-\hat{f}(k)\right]-\left[-A D_{0} \hat{x}(0)-G_{0} \hat{x}(0)-\hat{f}(0)\right]=0 \\
\Leftrightarrow i k D_{k} \hat{x}(k)-A D_{k} \hat{x}(k)-G_{k} \hat{x}(k)-\hat{f}(k)=0 \\
\Leftrightarrow\left(i k D_{k}-A D_{k}-G_{k}\right) \hat{x}(k)=\hat{f}(k) .
\end{gathered}
$$

Corollary 3.1 Assume that A generates a $C_{0}$-semigroup $(T(t))_{t \geq 0}$ on $X$ and let $f \in L^{p}(\mathbb{T} ; X), \quad 1 \leq p<\infty$ and $x$ be a mild solution of Equation (2). If $\left(i k D_{k}-A D_{k}-G_{k}\right)$ has a bounded inverse. Then

$$
\left(i k D_{k}-A D_{k}-G_{k}\right)^{-1} \text { is an } L^{p} \text {-multiplier. }
$$

Proof. From Theorem (3.2), we have that

$$
\hat{x}(k)=\left(i k D_{k}-A D_{k}-G_{k}\right)^{-1} \hat{f}(k) \text { for all } f \in L^{p}(\mathbb{T} ; X) .
$$

Our main result in this work is to establish that the converse of Theorem 3.1 and Corollary 3.1 are true, provided $X$ is an UMD space.

Theorem 3.3 Let $X$ be an UMD space and $A: D(A) \subset X \rightarrow X$ be an closed linear operator. Then the following assertions are equivalent for $1<p<\infty$.

1) for every $f \in L^{p}(\mathbb{T} ; X)$ there exists a unique $2 \pi$-periodic strong $L^{p}$-solution of Equation (2).

2) $\sigma_{\mathbb{Z}}(\Delta)=\phi$ and $\left\{i k \Delta_{k}^{-1}\right\}_{k \in \mathbb{Z}}$ is $R$-bounded.

Lemma 3.9 [1] Let $f, g \in L^{p}(\mathbb{T} ; X)$. If $\hat{f}(k) \in D(A)$ and $A \hat{f}(k)=\hat{g}(k)$ for all $k \in \mathbb{Z}$ Then

$$
f(t) \in D(A) \text { and } A f(t)=g(t) \text { for all } t \in[0,2 \pi]
$$




\section{Proof of Theorem 3.3:}

1) $\Rightarrow$ 2) see Theorem 3.1

$1) \Leftarrow 2)$ Let $f \in L^{p}(\mathbb{T} ; X)$. Define $\Delta_{k}=\left(i k D_{k}-A D_{k}-G_{k}\right)$.

By proposition 3.1, the family $\left\{i k \Delta_{k}^{-1}\right\}_{k \in \mathbb{Z}}$ is an $L^{p}$-multiplier it is equivalent to the family $\left\{\Delta_{k}^{-1}\right\}_{k \in \mathbb{Z}}$ is an $L^{p}$-multiplier that maps $L^{p}(\mathbb{T} ; X)$ into $H^{1, p}(\mathbb{T} ; X)$, namely there exists $x \in H^{1, p}(\mathbb{T}, X)$ such that

$$
\hat{x}(k)=\Delta_{k}^{-1} \hat{f}(k)=\left(i k D_{k}-A D_{k}-G_{k}\right)^{-1} \hat{f}(k) .
$$

In particular, $x \in L^{p}(\mathbb{T} ; X)$ and there exists $v \in L^{p}(\mathbb{T} ; X)$ such that $\hat{v}(k)=i k \hat{x}(k)$

$$
\widehat{(D x)^{\prime}}(k):=D_{k} \hat{v}(k)=i k D_{k} \hat{x}(k) .
$$

By Theorem 2.2, we have

$$
x_{t}(\theta)=x(t+\theta)=\lim _{n \rightarrow+\infty} \frac{1}{n+1} \sum_{m=0}^{n} \sum_{k=-m}^{m} \mathrm{e}^{i k t} \mathrm{e}^{i k \theta} \hat{x}(k) .
$$

Hence in $L^{p}(\mathbb{T} ; X)$, we obtain that

$$
x_{t}=\lim _{n \rightarrow+\infty} \frac{1}{n+1} \sum_{m=0}^{n} \sum_{k=-m}^{m} \mathrm{e}^{i k t} \mathrm{e}^{i k \cdot \hat{x}}(k) .
$$

Since $G$ is bounded, then

$$
\begin{aligned}
G x_{t} & =\lim _{n \rightarrow+\infty} \frac{1}{n+1} \sum_{m=0}^{n} \sum_{k=-m}^{m} \mathrm{e}^{i k t} G\left(e_{k} \hat{x}(k)\right) \\
& =\lim _{n \rightarrow+\infty} \frac{1}{n+1} \sum_{m=0}^{n} \sum_{k=-m}^{m} \mathrm{e}^{i k t} G_{k} \hat{x}(k) .
\end{aligned}
$$

Using now (3) and (4) we have:

$$
\widehat{(D x)^{\prime}}(k)=i k D_{k} \hat{x}(k)=A D_{k} \hat{x}(k)+G_{k} \hat{x}(k)+\hat{f}(k) \text { for all } k \in \mathbb{Z} \text {. }
$$

Since $A$ is closed, then $D x_{t} \in D(A)$ [Lemma 4.1] and from the uniqueness theorem of Fourier coefficients, that Equation (2) is valid.

Theorem 3.4 Let $1 \leq p<\infty$. Assume that A generates a $C_{0}$-semigroup $(T(t))_{t \geq 0}$ on $X$. If $\sigma_{Z}(\Delta)=\phi$ and $\left(i k D_{k}-A D_{k}-G_{k}\right)^{-1}$ is an $L^{p}$-multiplier Then there exists a unique mild periodic solution of Equation (2).

Proof. For $f \in L^{p}(\mathbb{T} ; X)$, we define

$$
f_{n}(t)=\frac{1}{n+1} \sum_{m=0}^{n} \sum_{k=-m}^{m} \mathrm{e}^{i k t} \hat{f}(k)
$$

By Theorem 2.2 we can assert that $f_{n} \rightarrow f$ as $n \rightarrow \infty$ for the norm in $L^{p}(\mathbb{T} ; X)$. We have $\left(i k D_{k}-A D_{k}-G_{k}\right)^{-1}$ is an $L^{p}$-multiplier then there exists $x \in L^{p}(\mathbb{T} ; X)$ such that

$$
\hat{x}(k)=\left(i k D_{k}-A D_{k}-G_{k}\right)^{-1} \hat{f}(k) .
$$

let

$$
x_{t, n}(\theta)=\frac{1}{n+1} \sum_{m=0}^{n} \sum_{k=-m}^{m} \mathrm{e}^{i k(t+\theta)}\left(i k D_{k}-A D_{k}-G_{k}\right)^{-1} \hat{f}(k) .
$$


Using again Theorem 2.2, we obtain that $x_{t, n} \rightarrow x_{t}(n \rightarrow \infty)$ and $x_{t, n}$ is strong $L^{p}$ solution of Equation (2) and $x_{t, n}$ verified

$$
D x_{t, n}=T(t) D \varphi_{n}+\int_{0}^{t} T(t-s) \int_{0}^{s}\left(G\left(\left(x_{n}\right)_{s}\right)+f_{n}(s)\right) \mathrm{d} s .
$$

let $y_{n}=D \varphi_{n}$. Then

$$
D x_{t, n}=T(t) y_{n}+\int_{0}^{t} T(t-s)\left(G\left(\left(x_{n}\right)_{s}\right)+f_{n}(s)\right) \mathrm{d} s .
$$

For $t=2 \pi$, we obtain that

$$
D x_{2 \pi, n}=T(2 \pi) y_{n}+\int_{0}^{2 \pi} T(2 \pi-s)\left(G\left(\left(x_{n}\right)_{s}\right)+f_{n}(s)\right) \mathrm{d} s .
$$

From which we infer that the sequence $\left(y_{n}\right)_{n}$ is convergent to some element $y$ as $n \rightarrow \infty$. Moreover, $y$ satisfies the following condition

$$
(*) y=T(2 \pi) y+\int_{0}^{2 \pi} T(2 \pi-s)\left(G\left(x_{s}\right)+f(s)\right) \mathrm{d} s
$$

let $n$ go to infinity in (5), we can write

$$
\begin{gathered}
D x_{t}=T(t) y+\int_{0}^{t} T(t-s)\left(G\left(x_{s}\right)+f(s)\right) \mathrm{d} s:=g(t) \\
g(2 \pi)=T(2 \pi) y+\int_{0}^{2 \pi} T(2 \pi-s)\left(G\left(x_{s}\right)+f(s)\right) \mathrm{d} s \stackrel{*}{=} y=g(0) .
\end{gathered}
$$

Then $D x_{2 \pi}=D \varphi \Rightarrow x_{2 \pi}=x_{0}$, we conclude that $x$ is a $2 \pi$-periodic mild solution of Equation (2).

\section{Applications}

Example 5.1: Let $A$ be a closed linear operator on a Hilbert space $H$ and suppose that $i \mathbb{Z} \subset \rho(A)$ and $\sup _{k \in \mathbb{Z}}\left\|k\left(i k D_{k}-A D_{k}\right)^{-1}\right\|=: M<\infty$.

If $\|G\|<\frac{1}{\left(2 r_{2 \pi}\right)^{1 / p} M}$ then for every $f \in L^{p}(\mathbb{T} ; X)$, there exists a unique strong $L^{p}$ solution of Equation (2).

From the identity

$$
i k D_{k}-A D_{k}-G_{k}=\left(i k D_{k}-A D_{k}\right)\left(I-G_{k}\left(i k D_{k}-A D_{k}\right)^{-1}\right)
$$

it follows that $i k D_{k}-A D_{k}-G_{k}$ is invertible whenever $\left\|G_{k}\left(i k D_{k}-A D_{k}\right)^{-1}\right\|<1$. [Theorem 2.3], we observe that $\left\|G_{k}\right\| \leq\left(2 r_{2 \pi}\right)^{1 / p}\|G\|$.

Hence,

$$
\left\|G_{k}\left(i k D_{k}-A D_{k}\right)^{-1}\right\|=\left\|G_{k}\left(i k D_{k}-A D_{k}\right)^{-1}\right\| \leq\left(2 r_{2 \pi}\right)^{1 / p}\|G\| M:=\alpha<1 .
$$

Then $\sigma_{Z}(\Delta)=\phi$ and by Theorem 2.3 we deduce that

$$
\begin{aligned}
\left(i k D_{k}-A D_{k}-G_{k}\right)^{-1} & =\left(i k D_{k}-A D_{k}\right)^{-1}\left(I-G_{k}\left(i k D_{k}-A D_{k}\right)^{-1}\right)^{-1} \\
& =\left(i k D_{k}-A D_{k}\right)^{-1} \sum_{n=0}^{\infty}\left[G_{k}\left(i k D_{k}-A D_{k}\right)^{-1}\right]^{n} .
\end{aligned}
$$




\section{Moreovery}

$$
\left\|i k\left(i k D_{k}-A D_{k}-G_{k}\right)^{-1}\right\| \leq\left\|i k\left(D_{k}-A D_{k}\right)\right\| \sum_{n=0}^{\infty}\left[G_{k}\left(i k D_{k}-A D_{k}\right)^{-1}\right]^{n} \leq \frac{1+M}{1-\alpha}
$$

and

$$
\sup _{k \in \mathbb{Z}}\left\|i k\left(i k D_{k}-A D_{k}-G_{k}\right)^{-1}\right\|<\infty .
$$

We conclude that there exists a unique strong $L^{p}$-solution of Equation (2). Using Corollary 3.8 in [2].

\section{Example 5.2:}

Let $A$ be a closed linear operator and $X$ be a Hilbert space such that $i \mathbb{Z} \subset \rho(A)$ and $R_{p}\left(k\left(i k D_{k}-A D_{k}\right)^{-1}\right)=: M<\infty$. Suppose that $\|G\|<\frac{1}{\left(2 r_{2 \pi}\right)^{1 / p} M}$. Then using Lemma 2.1

(1), we obtain that

$$
\sup _{k \in \mathbb{Z}}\left\|k\left(i k D_{k}-A D_{k}-G_{k}\right)^{-1}\right\| \leq R_{p}\left(k\left(i k D_{k}-A D_{k}\right)^{-1}\right)=M .
$$

From the identity $i k D_{k}-A D_{k}-G_{k}=\left(i k D_{k}-A D_{k}\right)\left(I-G_{k}\left(i k D_{k}-A D_{k}\right)^{-1}\right)$ it follows that $i k D_{k}-A D_{k}-G_{k}$ is invertible whenever

$$
\left\|G_{k}\left(i k D_{k}-A D_{k}\right)^{-1}\right\|<1 .
$$

Observe that $\left\|G_{k}\right\| \leq\left(2 r_{2 \pi}\right)^{1 / p}\|G\|$.

Hence

$$
\left\|G_{k}\left(i k D_{k}-A D_{k}\right)^{-1}\right\|=\left\|G_{k}\left(i k D_{k}-A D_{k}\right)^{-1}\right\| \leq\left(2 r_{2 \pi}\right)^{1 / p}\|G\| M=\alpha<1 .
$$

Then $\sigma_{Z}(\Delta)=\phi$ and by Theorem 2.3, we have

$$
\begin{aligned}
& \left(i k D_{k}-A D_{k}-G_{k}\right)^{-1}=\left(i k D_{k}-A D_{k}\right)^{-1}\left(I-G_{k}\left(i k D_{k}-A D_{k}\right)^{-1}\right)^{-1} \\
& =\left(i k D_{k}-A D_{k}\right)^{-1} \sum_{n=0}^{\infty}\left[G_{k}\left(i k D_{k}-A D_{k}\right)^{-1}\right]^{n} . \\
& R_{p}\left(i k\left(i k D_{k}-A D_{k}\right)^{-1}\left[G_{k}\left(i k D_{k}-A D_{k}\right)^{-1}\right]^{n}\right) \\
& \leq R_{p}\left(i k\left(i k D_{k}-A D_{k}\right)^{-1}\right)\left[R_{p}\left(G_{k}\left(i k D_{k}-A D_{k}\right)^{-1}\right)\right]^{n} \\
& \leq R_{p}\left(i k\left(i k D_{k}-A D_{k}\right)^{-1}\right)\left[R_{p}\left(G_{k}\right)\right]^{n}\left[R_{p}\left(\left(i k D_{k}-A D_{k}\right)^{-1}\right)\right]^{n} \\
& \leq R_{p}\left(i k\left(i k D_{k}-A D_{k}\right)^{-1}\right)\left(\left(2 r_{2 \pi}\right)^{1 / p}\|G\|\right)^{n} M^{n} \\
& =R_{p}\left(i k\left(i k D_{k}-A D_{k}\right)^{-1}\right) \alpha^{n} .
\end{aligned}
$$

Finaly

$$
R_{p}\left(\left(i k D_{k}-A D_{k}-G_{k}\right)^{-1}\right) \leq R_{p}\left(i k\left(i k D_{k}-A D_{k}\right)^{-1}\right) \frac{1}{1-\alpha} \leq \frac{M+1}{1-\alpha} .
$$

This proves that $\left\{i k\left(D_{k}-A D_{k}-G_{k}\right)^{-1}\right\}$ is $R$-bounded and by Theorem 3.3, we get 
that there exists a unique strong $L^{p}$-solution of (2).

\section{Acknowledgements}

The authors would like to thank the referee for his remarks to improve the original version.

\section{References}

[1] Arend, W. and Bu, S. (2002) The Operator-Valued Marcinkiewicz Multiplier Theorem and Maximal Regularity. Mathematische Zeitschrift, 240, 311-343.

http://dx.doi.org/10.1007/s002090100384

[2] Lizama, C. (2006) Fourier Multipliers and Perodic Solutions of Delay Equatons in Banach Spaces. Journal of Mathematical Analysis and Applications, 324, 921-933. http://dx.doi.org/10.1016/j.jmaa.2005.12.043

[3] Henriquez, H.R., Pierri, M. and Prokopczyk, A. (2012) Periodic Solutions of Abstract Neutral Functional Differential Equations. Journal of Mathematical Analysis and Applications, 385, 608-621. http://dx.doi.org/10.1016/j.jmaa.2011.06.078

[4] Benkhalti, R., Bouzahir, H. and Ezzinbi, K. (2001) Existence of a Periodic Solution for Some Partial Functional Differential Equations with Infinite Delay. Journal of Mathematical Analysis and Applications, 256, 257-280. http://dx.doi.org/10.1006/jmaa.2000.7321

[5] Adimy, M., Bouzahir, H. and Ezzinbi, K. (2004) Existence and Stability for Some Partial Nentral Functional Differential Equations with Infinite Delay. Journal of Mathematical Analysis and Applications, 294, 438-461. http://dx.doi.org/10.1016/j.jmaa.2004.02.033

[6] Adimy, M., Ezzinbi, K. and Laklach, M. (2000) Local Existence and Global Continuation for a Class of Partial Neutral Functional Differential Equations. Comptes Rendus de 1 Académie des Sciences-Series I-Mathematics, 330, 957-962.

[7] Adimy, M. and Ezzinbi, K. (1998) A Class of Linear Partial Neutral Functional Differential Equations with Non-Dense Domain. Journal of Differential Equations, 147, 285-332. http://dx.doi.org/10.1006/jdeq.1998.3446

[8] Adimy, M and Ezzinbi, K. (1998) Local Existence and Linearized Stability for Partial Functional Differential Equations. Dynamic Systems and Applications, 7, 389-404.

[9] Bourgain, J. (1983) Some Remarks on Banach Spaces in Which Martingale Differences Sequences Are Unconditional. Arkiv för Matematik, 21, 163-168.

http://dx.doi.org/10.1007/BF02384306 
Submit or recommend next manuscript to SCIRP and we will provide best service for you:

Accepting pre-submission inquiries through Email, Facebook, LinkedIn, Twitter, etc. A wide selection of journals (inclusive of 9 subjects, more than 200 journals)

Providing 24-hour high-quality service

User-friendly online submission system

Fair and swift peer-review system

Efficient typesetting and proofreading procedure

Display of the result of downloads and visits, as well as the number of cited articles

Maximum dissemination of your research work

Submit your manuscript at: http://papersubmission.scirp.org/

Or contactapm@scirp.org 